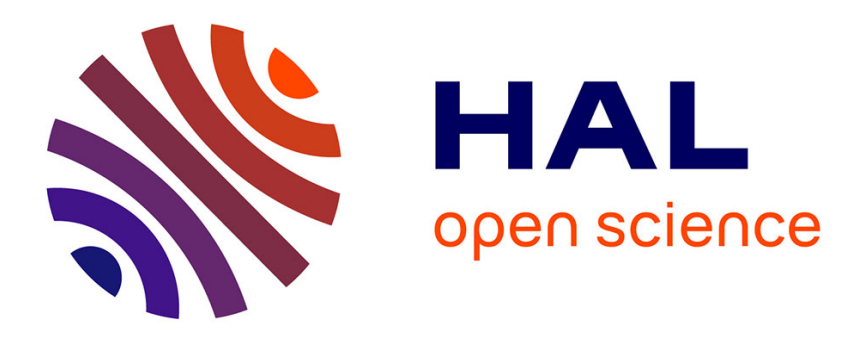

\title{
Maximal Supersymmetry and Non-Abelian Gauge Groups
}

Henning Samtleben

\section{To cite this version:}

Henning Samtleben. Maximal Supersymmetry and Non-Abelian Gauge Groups. XVIth International Congress on Mathematical Physics, Prague, Czech Republic. ensl-00491052

\section{HAL Id: ensl-00491052 \\ https://hal-ens-lyon.archives-ouvertes.fr/ensl-00491052}

Submitted on 10 Jun 2010

HAL is a multi-disciplinary open access archive for the deposit and dissemination of scientific research documents, whether they are published or not. The documents may come from teaching and research institutions in France or abroad, or from public or private research centers.
L'archive ouverte pluridisciplinaire $\mathbf{H A L}$, est destinée au dépôt et à la diffusion de documents scientifiques de niveau recherche, publiés ou non, émanant des établissements d'enseignement et de recherche français ou étrangers, des laboratoires publics ou privés. 


\title{
MAXIMAL SUPERSYMMETRY AND NON-ABELIAN GAUGE GROUPS ${ }^{1}$
}

\author{
Henning Samtleben \\ Université de Lyon, Laboratoire de Physique, \\ UMR 5672, CNRS et ENS de Lyon, \\ 46 allée d'Italie, F-69364 Lyon CEDEX 07, France \\ henning.samtleben @ens-lyon.fr
}

\begin{abstract}
I review recent progress in the construction and classification of maximally supersymmetric theories with non-abelian gauge groups. The algebraic framework is based on the underlying exceptional symmetry groups. This has applications for supergravity theories describing flux compactifications as well as for the recently constructed three-dimensional superconformal theories describing the dynamics of multiple M2-branes.
\end{abstract}

\section{Introduction}

Supersymmetric theories in four dimensions are invariant under symmetries that close into the superextension of the Poincaré algebra

$$
\left\{Q_{\alpha}^{i}, \bar{Q}_{j \beta}\right\}=\delta_{j}^{i} \gamma_{\alpha \beta}^{\mu} P_{\mu},
$$

where the indices $\mu$ and $\alpha, \beta$ refer to space-time vectors and spinors, respectively. The indices $i, j=1, \ldots, N$ label the number of supercharges. As a result, the field content of these theories falls into representations of the superalgebra (1). Algebraic consistency restricts the maximal number of supercharges to $N=4$ and $N=8$ for global and local supersymmetry, respectively. The resulting supermultiplets are collected in table 1 , the states being organized by the helicity group $S O(2)$.

\footnotetext{
${ }^{1}$ To appear in the proceedings of the 16th International Congress on Mathematical Physics, Prague, August 3-8, 2009.
} 


\begin{tabular}{c|rrrrrrrrr}
\hline helicity & -2 & $-\frac{3}{2}$ & -1 & $-\frac{1}{2}$ & 0 & $+\frac{1}{2}$ & +1 & $+\frac{3}{2}$ & +2 \\
\hline$N=4:$ & & & 1 & 4 & 6 & 4 & 1 & & \\
\hline$N=8:$ & 1 & 8 & 28 & 56 & 70 & 56 & 28 & 8 & 1 \\
\hline
\end{tabular}

Table 1: Maximal supermultiplets.

The $N=4$ supermultiplet combines 6 scalar fields with their fermionic superpartners and the two helicity states of a massless vector field. The most general theory with global $N=4$ supersymmetry combines an arbitrary number $n$ of these multiplets, whose gauge fields realize a gauge group $G$ of dimension $n$. These are the super Yang-Mills theories [1]. Their classification thus reduces to the classification of Lie algebras $\mathfrak{g}$ of a given dimension, i.e. to the solution of the Jacobi identities

$$
f_{[a b}^{c} f_{d] c}^{e}=0 .
$$

The situation is quite different for maximal local supersymmetry, i.e. for theories realizing the $N=8$ supermultiplet of table 1 . As this multiplet includes the spin- 2 states of the space-time metric, only a single copy can be described by an interacting theory. It then becomes a non-trivial question which gauge groups can be realized by the 28 gauge fields while preserving maximal local supersymmetry. Indeed, after the original construction of $N=8$ supergravity [2] with abelian gauge group $U(1)^{28}$ and the first nonabelian version [3] with gauge group $S O(8)$, a variety of other viable noncompact and non-semisimple gauge groups were discovered $[4,5,6]$. A convenient framework to achieve a general classification uses the so-called embedding tensor $[7,8,9]$. In the following I will review this formalism which

- generalizing (2) - reduces the classification of maximal supergravities to a set of algebraic equations for the embedding tensor. I sketch the emerging structure of non-abelian tensor fields in these theories and finish with an example of a non-trivial application of the formalism also in theories with global supersymmetry.

\section{Gauged supergravity}

A distinguished property of the abelian $N=8$ supergravity is the underlying global symmetry group $G_{0}=E_{7(7)}[2]$. Its 70 scalar fields parametrize the coset space manifold $E_{7(7)} / S U(8)$ while its 28 vector fields combine with their magnetic duals into the fundamental 56-dimensional representation of 
$E_{7(7)}$, which we denote by $A_{\mu}^{M},(M=1, \ldots, 56)$. On the physical fields of table 1 , this symmetry thus acts in a non-linear and non-local way; infinitesimally, we parametrize this action as

$$
\delta_{\Lambda} \Phi=\Lambda^{\alpha} t_{\alpha} \cdot \Phi
$$

for an arbitrary physical field $\Phi$, parameter $\Lambda$ and with $t_{\alpha}$ denoting the generators of $\mathfrak{e}_{7(7)}=\operatorname{Lie} E_{7(7)}$ (i.e. $\alpha=1, \ldots, 133$ parametrizing the adjoint representation), closing into

$$
\left[t_{\alpha}, t_{\beta}\right]=f_{\alpha \beta}^{\gamma} t_{\gamma} .
$$

In this version of the theory, none of the matter fields is charged under the $U(1)^{28}$ gauge group. Non-abelian deformations of the theory are obtained by selecting a subset of generators $t_{\alpha}$ and promoting their action (3) into a local symmetry. The choice of generators is conveniently parametrized as

$$
X_{M} \equiv \Theta_{M}^{\alpha} t_{\alpha}
$$

by a constant embedding tensor $\Theta_{M}{ }^{\alpha}$, which encodes the embedding of the gauge group into the global $E_{7(7)}$ of the ungauged theory. The action of the generators $X_{M}$ can be promoted to a local symmetry by introducing standard covariant derivatives ${ }^{2}$

$$
D_{\mu} \equiv \partial_{\mu}+A_{\mu}^{M} X_{M}
$$

Not every choice of generators (5) leads to a consistent theory. In particular, the generators $X_{M}$ must close into a subalgebra. Moreover, it is only for very particular subalgebras that the deformation is compatible with maximal supersymmetry. Fortunately, all consistency conditions translate into a simple set of algebraic equations for the embedding tensor $\Theta_{M}{ }^{\alpha}$. Closure of the gauge algebra requires

$$
\Theta_{P}^{\beta} \Theta_{N}^{\alpha}\left(t_{\beta}\right)_{M}^{N}+\Theta_{P}^{\beta} \Theta_{M}^{\gamma} f_{\beta \gamma}^{\alpha}=0,
$$

which can be interpreted as generalization of the Jacobi identities (2). Supersymmetry imposes a linear constraint on the embedding tensor

$$
\Theta_{M}^{\alpha}=\left(\mathbb{P}_{912} \Theta\right)_{M}^{\alpha}
$$

\footnotetext{
${ }^{2}$ To be as general as possible, we allow for connections combining electric and magnetic gauge fields. It turns out that for consistent choice of $\Theta_{M}{ }^{\alpha}$ (see below) this gives rise to sensible theories which allow for an off-shell formulation even in the presence of magnetic charges [10].
} 
where the projector refers to the tensor product decomposition

$$
56 \otimes 133=56 \oplus 912 \oplus 6480,
$$

of the fundamental and the adjoint representation of $E_{7(7)}$, corresponding to the two indices of the embedding tensor. This leaves 912 free parameters in $\Theta_{M}{ }^{\alpha}$, subject to the 8778 quadratic equations (7). It can be shown [9] that every solution to the system of algebraic equations (7), (8) defines a consistent theory with maximal local supersymmetry and gauge group spanned by the generators (5). In particular, the scalar potential that appears in the gauged theory, takes the form

$$
\begin{aligned}
V & =\Theta_{M}{ }^{\alpha} \Theta_{N}{ }^{\beta} V^{M N}{ }_{\alpha \beta}(\phi), \\
V^{M N}{ }_{\alpha \beta}(\phi) & \equiv\left(\left(t_{\alpha}\right)_{P}{ }^{R}\left(t_{\beta}\right)_{Q}{ }^{S} \mathcal{M}^{P Q} \mathcal{M}_{R S}+7\left(t_{\alpha}\right)_{P}{ }^{Q}\left(t_{\beta}\right)_{Q}{ }^{P}\right) \mathcal{M}^{M N},
\end{aligned}
$$

bilinear in the embedding tensor $\Theta_{M}^{\alpha}$. The scalar dependence arises through the symmetric positive definite matrices $\mathcal{M}=\mathcal{V} \mathcal{V}^{T}$, with $\mathcal{V}$ denoting the $E_{7(7)} / S U(8)$ coset representative, see [9] for details.

The universal form of the Lagrangian of the gauged theory and in particular of its scalar potential (10) is of particular importance for the study of the low energy effective actions associated to particular flux compactifications of higher dimensional theories. E.g. in flux compactifications of IIA/IIB supergravity on (twisted) tori, the flux parameters show up as deformation parameters among the components of the embedding tensor and can be uniquely identified by purely group-theoretical means, see [11] and references therein. The expression (10) then allows to read off the full scalar potential of the effective theory upon specifying the particular choice of $\Theta_{M}{ }^{\alpha}$.

The framework sketched here can equally well be applied to the construction of gauged supergravity theories in other space-time dimensions and with a lower number of supersymmetries, in which case the constraints (7), (8) are replaced by equations covariant under the relevant global symmetry group.

\section{Non-abelian tensor fields}

Apart from the minimal couplings induced by the covariant derivatives (6), the field strengths of the vector fields acquire the standard non-abelian modification

$$
\mathcal{F}_{\mu \nu}^{M}=\partial_{\mu} A_{\nu}^{M}-\partial_{\nu} A_{\mu}^{M}+X_{[N P]}^{M} A_{\mu}^{N} A_{\nu}^{P},
$$

with $X_{M N}{ }^{P} \equiv \Theta_{M}{ }^{\alpha} t_{\alpha N}{ }^{P}$. While in standard Yang-Mills theory this defines covariant objects, here the object (11) in general does not transform covariantly under local gauge transformations. Again, the reason is, that vector 
fields a priori transform in a given fundamental representation of the global symmetry group; as a consequence, the "structure constants" $X_{M N}{ }^{P}$ do not satisfy the standard Jacobi identities. The natural covariant object turns out to be the following combination [9]

$$
\mathcal{H}_{\mu \nu}^{M}=\mathcal{F}_{\mu \nu}^{M}+Z^{M \alpha} B_{\mu \nu \alpha},
$$

with $Z^{M \alpha} \equiv-\frac{1}{2} \Omega^{M N} \Theta_{N}{ }^{\alpha}$, upon the introduction of two-form tensor fields $B_{\mu \nu \alpha}$, transforming in the adjoint representation of $E_{7(7)}$. The new field strength $\mathcal{H}_{\mu \nu}^{M}$ transforms covariantly under the combined set of gauge transformations

$$
\begin{aligned}
\delta A_{\mu}^{M} & =D_{\mu} \Lambda^{M}-Z^{M \alpha} \Xi_{\mu \alpha}, \\
\delta B_{\mu \nu \alpha} & =2 D_{[\mu} \Xi_{\nu] \alpha}+2\left(t_{\alpha}\right)_{M}{ }^{K} \Omega_{K N}\left(\Lambda^{M} \mathcal{H}_{\mu \nu}^{N}-A_{[\mu}^{M} \delta A_{\nu]}^{N}\right),
\end{aligned}
$$

which exhibit Stückelberg-type couplings between vector fields and antisymmetric two-forms, as is familiar from massive deformations of supergravities, e.g. [12].

Similarly, the construction of a covariant field strength for the two-forms requires the introduction of three-form tensor fields, etc. The final result is a full hierarchy of non-abelian tensor fields which (schematically) extends (13) to

$$
\begin{aligned}
\delta B_{\mu \nu} & =2 D_{[\mu} \Xi_{\nu]}+\ldots-Y \Phi_{\mu \nu}, \\
\delta C_{\mu \nu \rho} & =3 D_{[\mu} \Phi_{\nu \rho]}+\ldots-W \Sigma_{\mu \nu \rho}, \\
\delta D_{\mu \nu \rho \sigma} & =4 D_{[\mu} \Sigma_{\nu \rho \sigma]}+\ldots
\end{aligned}
$$

where we have omitted all $E_{7(7)}$-indices, see $[13,14]$ for details. The intertwining tensors $Z, Y$ and $W$ are functions of the embedding tensor and determine the precise $E_{7(7)}$ representation content in which the higher rank $p$-forms must appear. For the $N=8$ theory, the required forms $B, C$ and $D$ transform in the $\mathbf{1 3 3}$, the $\mathbf{9 1 2}$, and the $\mathbf{1 3 3}+\mathbf{8 6 4 5}$, respectively. This gives agreement with the predictions obtained from analyzing the branchings of the infinite-dimensional representations of the underlying very extended Kac-Moody algebra [15, 16]. Moreover, this representation assignment indicates that the higher rank forms do not represent new propagating degrees of freedom but magnetic duals to the physical field content: the two-forms are dual to the $133 E_{7(7)}$ Noether currents, while the 912 three-forms are related to the deformation parameters combined in the embedding tensor as

$$
D_{[\mu} C_{\nu \rho \sigma]}{ }^{M}{ }_{\alpha}+\ldots=V^{M N}{ }_{\alpha \beta}(\phi) \Theta_{N}{ }^{\beta},
$$


with the scalar dependent matrix $V^{M N}{ }_{\alpha \beta}(\phi)$ from (10). The 8778 fourforms $D$ in turn can be interpreted as Lagrange multipliers for the quadratic constraints (7) [13].

\section{Global supersymmetry in three dimensions}

In certain cases, the embedding tensor formalism also provides a valuable framework for theories with global supersymmetry [17, 18]. In three dimensions, maximally supersymmetric Yang-Mills theory is obtained by dimensional reduction from ten dimensions [1]. However, another $N=8$ theory can be constructed by gauging the free theory of $n$ scalar multiplets $\left\{\Phi^{I k}, \Psi^{\dot{A} k}\right\}$, labeled by $k$, with $I$ and $\dot{A}$ labeling $S O(8)$ vectors and spinors, respectively. The $S O(n)$ Noether currents

$$
J_{\mu}^{k l}=\Phi^{I[k} \partial_{\mu} \Phi^{l] I}+\bar{\Psi}^{\dot{A}[k} \gamma_{\mu} \Psi^{l] \dot{A}},
$$

allow for the introduction of dual vector fields which can be used to gauge (a subgroup of) the global $S O(n)$ group. In three dimensions, the embedding tensor thus is of the form $\Theta_{M}{ }^{\alpha} \rightarrow \Theta_{k l, m n}$. The constraints (7), (8) in this case take the form

$$
\begin{aligned}
\Theta_{k l, m n} & =\Theta_{[k l, m n]}, \\
0 & =\Theta_{k l, p r} \Theta_{m n, r q}-\Theta_{m n, p r} \Theta_{k l, r q}-\Theta_{k l, m r} \Theta_{n r, p q}+\Theta_{k l, n r} \Theta_{m r, p q}
\end{aligned}
$$

which reproduces the equations underlying the three-algebra structure of Bagger and Lambert [19], to which $S O(4)$ is the only solution with $\Theta_{k l, m n}=$ $\epsilon_{k l m n}$. For $N<8$, in contrast the corresponding equations admit a variety of possible solutions.

\section{Acknowledgments}

I am most grateful to E. Bergshoeff, B. de Wit, O. Hohm, A. Le Diffon, H. Nicolai, D. Roest, E. Sezgin and M. Trigiante for many discussions and collaboration on the topics presented. This work is supported by the Agence Nationale de la Recherche.

\section{References}

[1] L. Brink, J. H. Schwarz and J. Scherk, Nucl. Phys. B121, 77 (1977). 
[2] E. Cremmer and B. Julia, Nucl. Phys. B159, 141 (1979).

[3] B. de Wit and H. Nicolai, Nucl. Phys. B208, 323 (1982).

[4] C. M. Hull, Phys. Lett. B142, 39 (1984).

[5] L. Andrianopoli, R. D'Auria, S. Ferrara and M. A. Lledo, JHEP 07, 010 (2002).

[6] C. M. Hull, Class. Quant. Grav. 20, 5407 (2003).

[7] H. Nicolai and H. Samtleben, Phys. Rev. Lett. 86, 1686 (2001).

[8] B. de Wit, H. Samtleben and M. Trigiante, Nucl. Phys. B655, 93 (2003).

[9] B. de Wit, H. Samtleben and M. Trigiante, JHEP 06, 049 (2007).

[10] B. de Wit, H. Samtleben and M. Trigiante, JHEP 09, 016 (2005).

[11] H. Samtleben, Class. Quant. Grav. 25, 214002 (2008).

[12] L. J. Romans, Phys. Lett. B169, 374 (1986).

[13] B. de Wit, H. Nicolai and H. Samtleben, JHEP 02, 044 (2008).

[14] E. Bergshoeff, J. Hartong, O. Hohm, M. Hübscher and T. Ortin, JHEP 04, 123 (2009).

[15] F. Riccioni and P. West, JHEP 07, 063 (2007).

[16] E. Bergshoeff, I. De Baetselier and T. A. Nutma, JHEP 09, 047 (2007).

[17] E. Bergshoeff, M. de Roo and O. Hohm, Class. Quant. Grav. 25, 142001 (2008).

[18] E. Bergshoeff, O. Hohm, D. Roest, H. Samtleben and E. Sezgin, JHEP 09, 101 (2008).

[19] J. Bagger and N. Lambert, Phys. Rev. D77, 065008 (2008). 